\title{
HEP ML/Optimization Go Quantum - QuantISED Pilot
}

Steven Adachi ${ }^{3}$, João Caldeira ${ }^{1}$, Andrea Delgado ${ }^{2}$, Kathleen Hamilton $^{2}$, Travis Humble ${ }^{2}$, Joshua Job ${ }^{3}$, James Kowalkowski ${ }^{1}$, Stefan $^{1}$ Leichenauer ${ }^{5}$, Alex McCaskey ${ }^{2}$, Stephen Mrenna ${ }^{1}$, Brian Nord ${ }^{1}$, Gabriel Perdue ${ }^{1}$, Evan Peters ${ }^{1,4}$, Aristeidis Tsaris $^{1}$

${ }^{1}$ Fermi National Accelerator Laboratory, ${ }^{2}$ Oak Ridge National Laboratory, ${ }^{3}$ Lockheed Martin, ${ }^{4}$ University of Waterloo, ${ }^{5}$ Alphabet's $X$

FERMILAB-POSTER-20-042-SCD-QIS

Quantum annealers for classification arxiv: 1911.06259

Use Restricted Boltzmann Machines for classification of galaxy morphology. To put data on the D-Wave, we need to compress images to a small number of bits. For this, we use Principal Component Analysis.
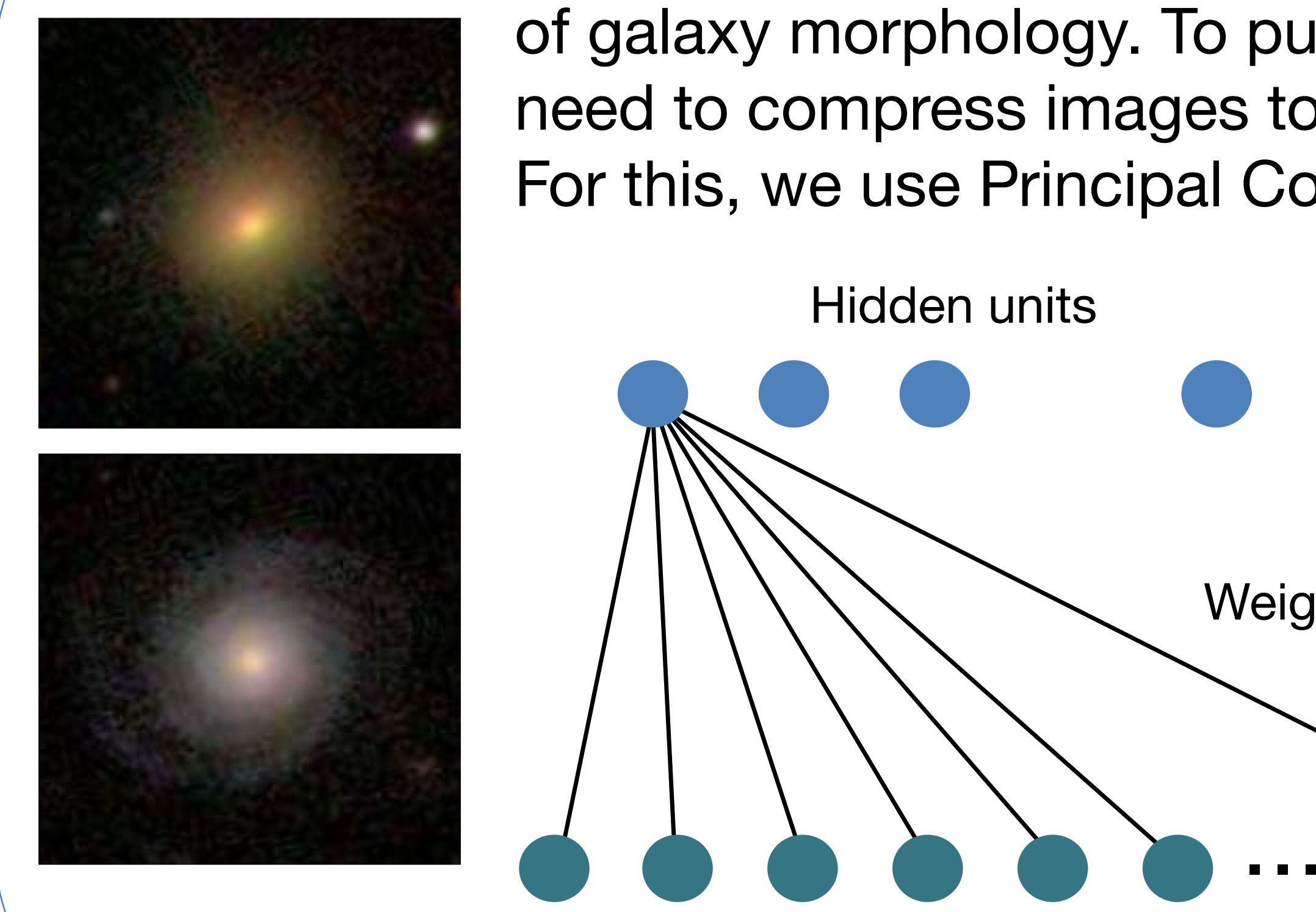

Visible units: compressed data

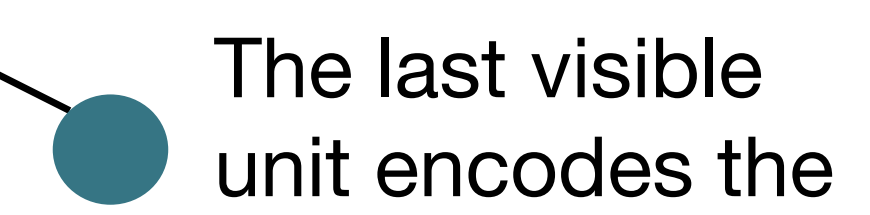
class of the class of the
object

Below, we show the accuracy achieved by RBMs hidden units, indicated below the graph).
Quantum-trained RBMs are competitive at early of different size (same number of visible and Quantum-trained RBMs are competitive at early
stages, but dominated by classical RBMs later.

\section{Software and Workflows}

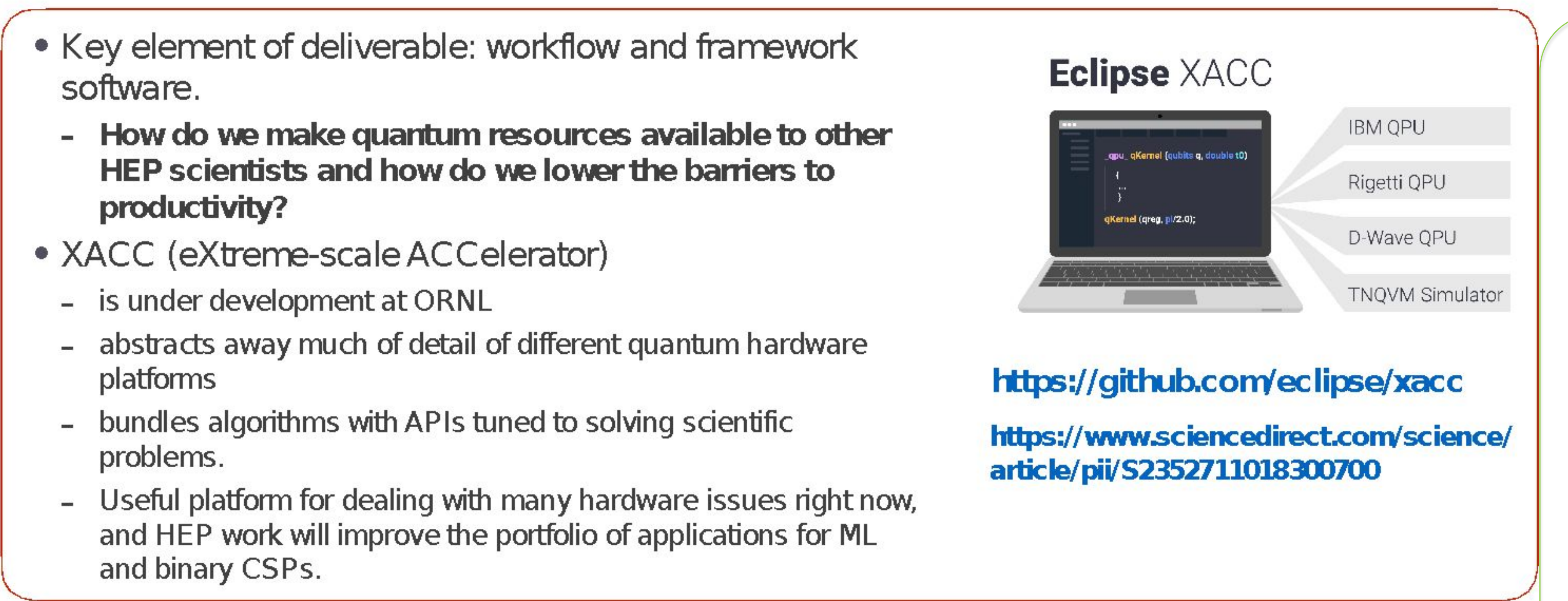

-XACC
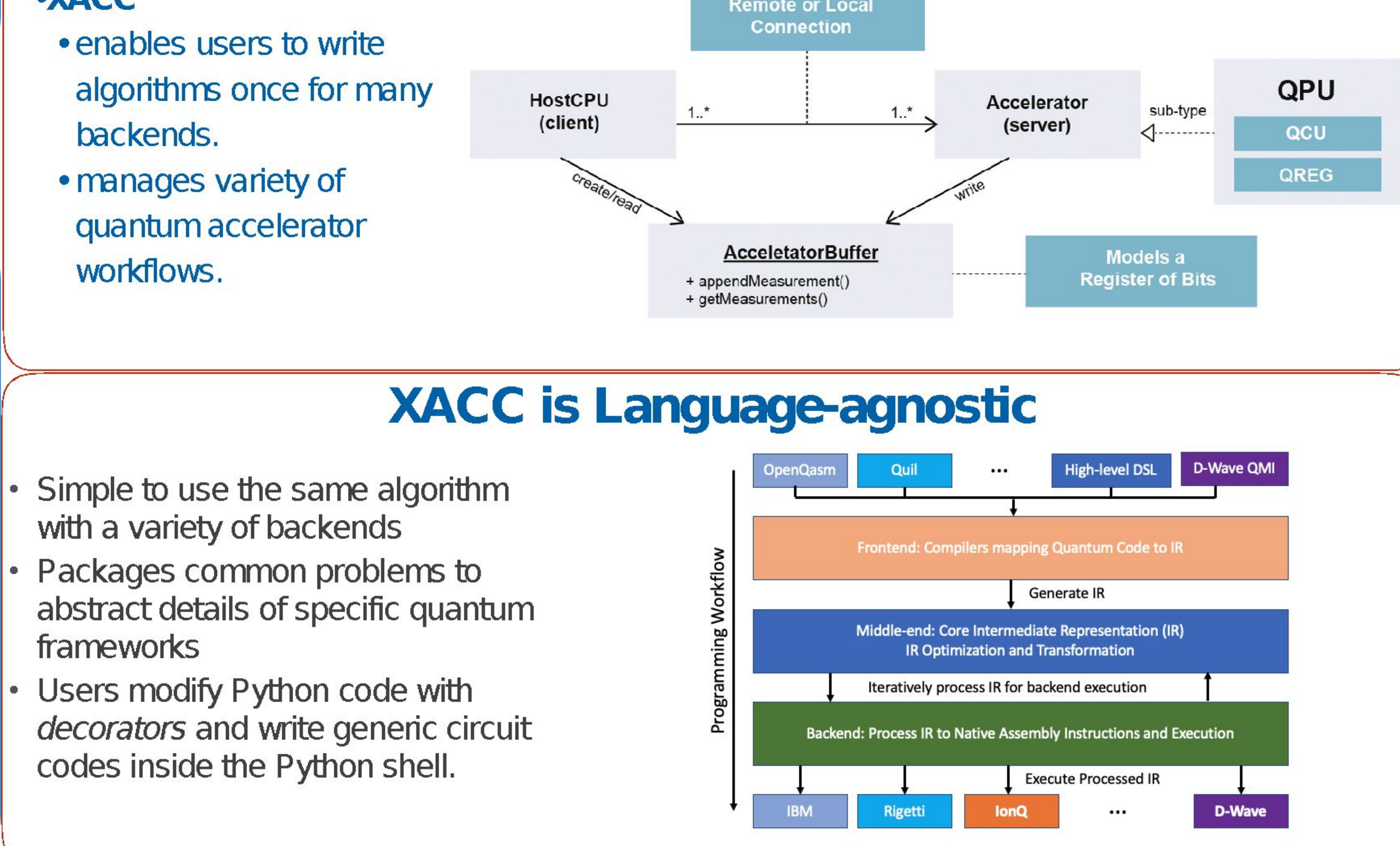

XACC is High-level

Makes it simple to run common

algorithms and applications with ven litte work

Data ingestion is simple and flexible

Details of hybrid quantum-classical programming are abstracted away. - Easy to extend when users want to run new algonthms.

Prototype of a complete $R B M$ classifier.

XACC and Hybrid Variational Algorithms

- Algorithm Interface

General protocol / data structure for describing hybrid workflows Initialize with problem-specific data

Execute, persist results to buffe

- Hardware Agnostic

easily swap between gate

model QCs from IBM and Rigetti

- Efficient observable definition

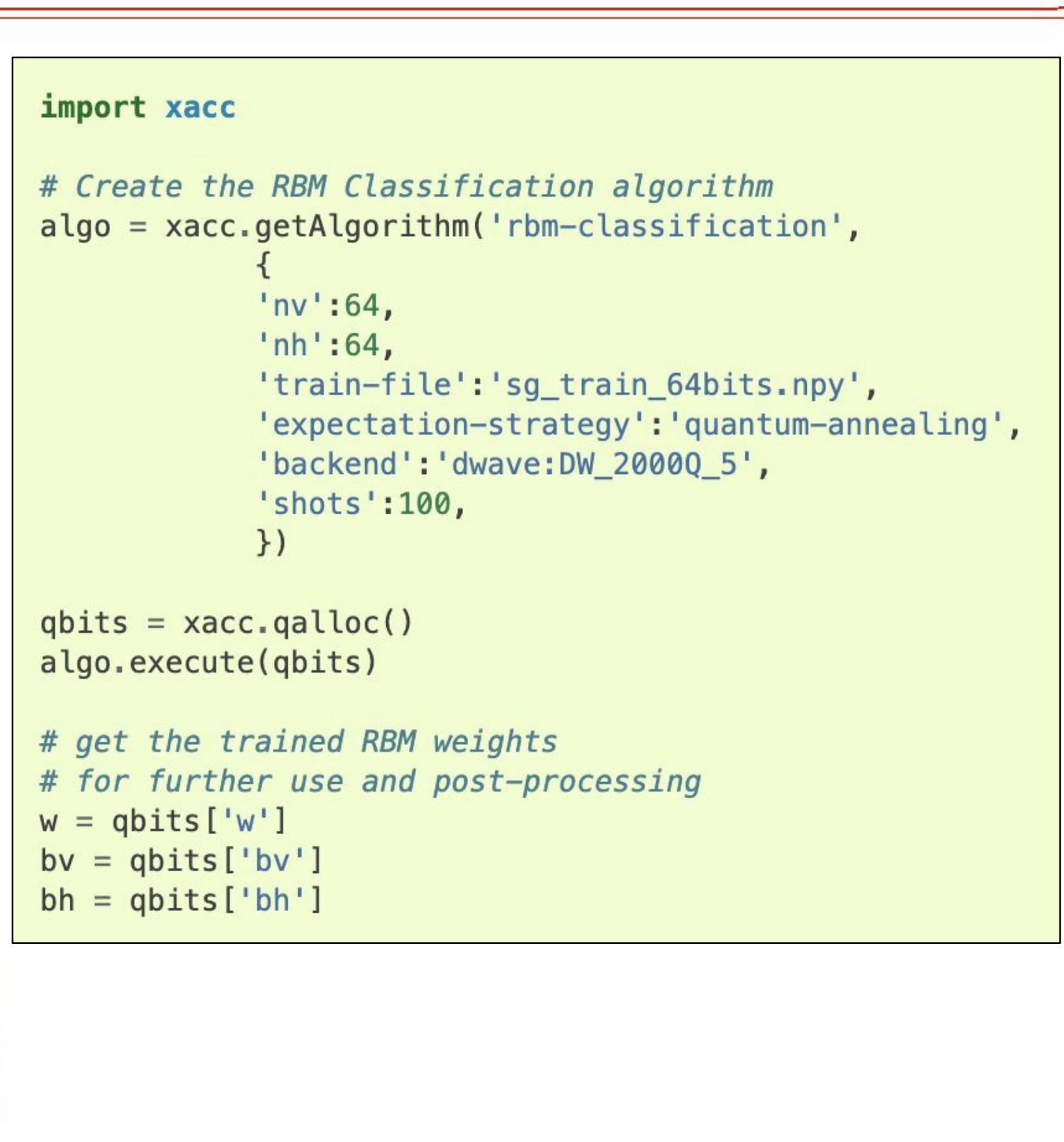

The plots show the $\mathrm{KL}$ divergence between the state distribution obtained on the hardware and that obtained on a noisy simulation for an 8-qubit circuit. The noise model is fairly accurate.

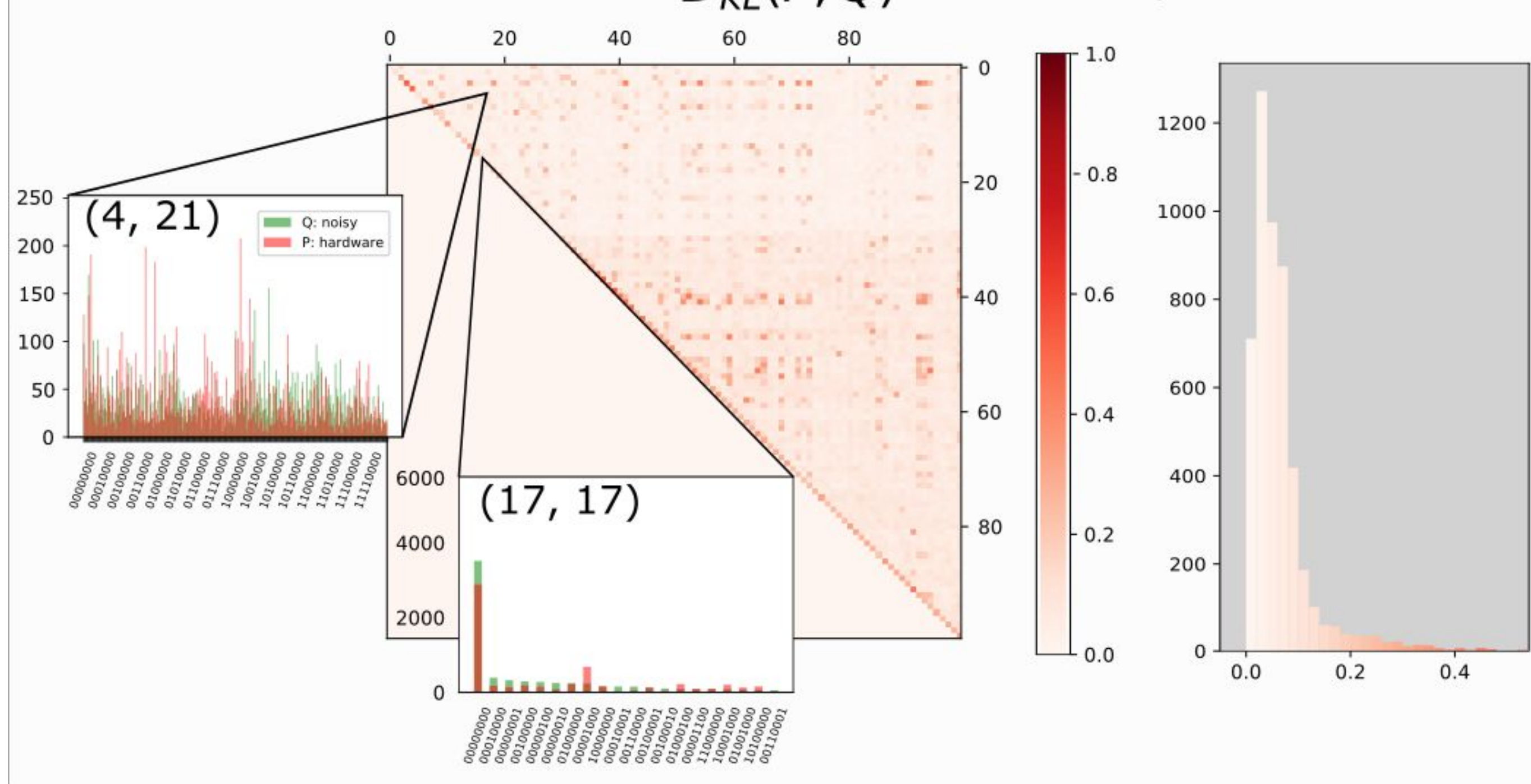

The Google Sycamore chip being used in the

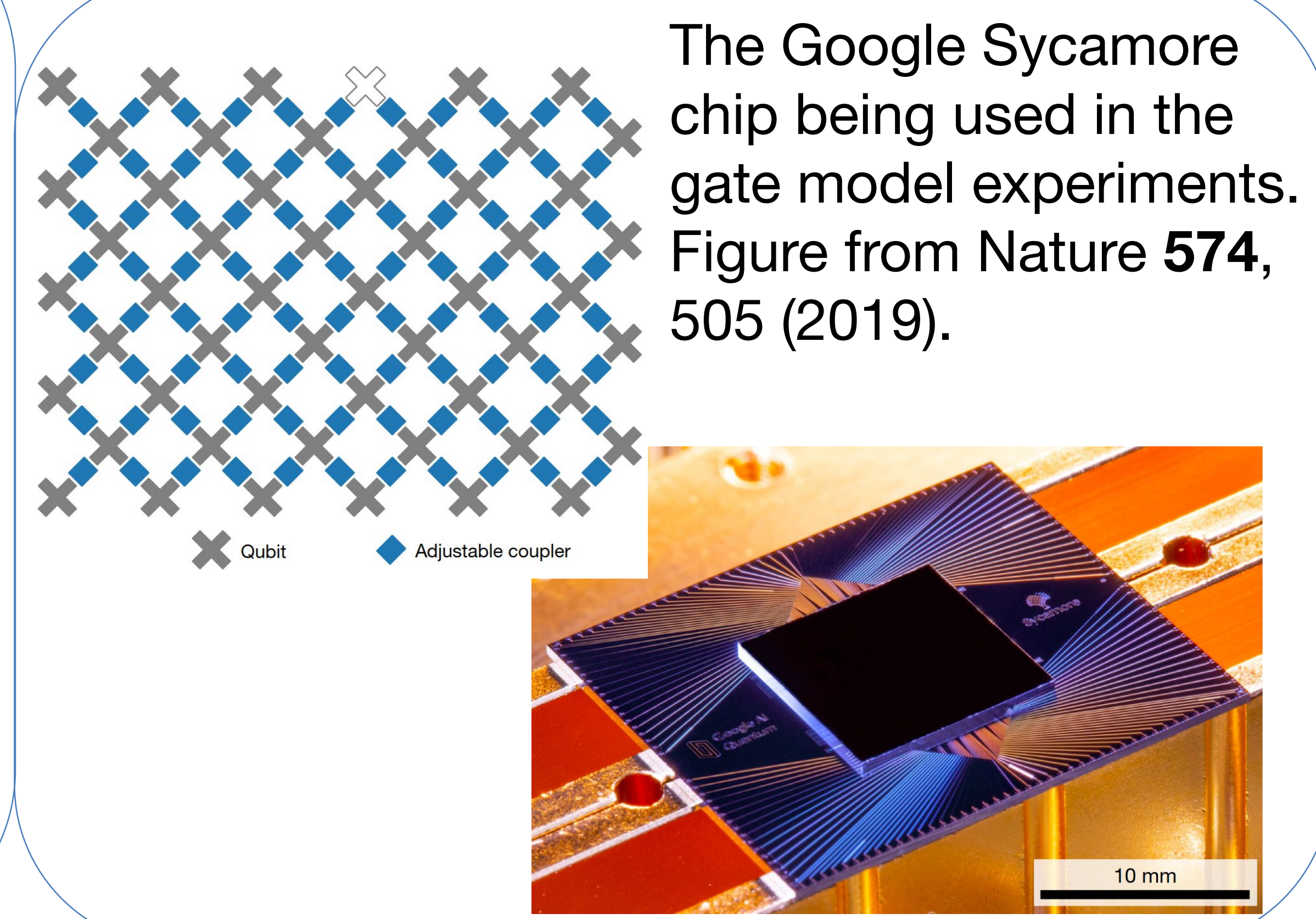

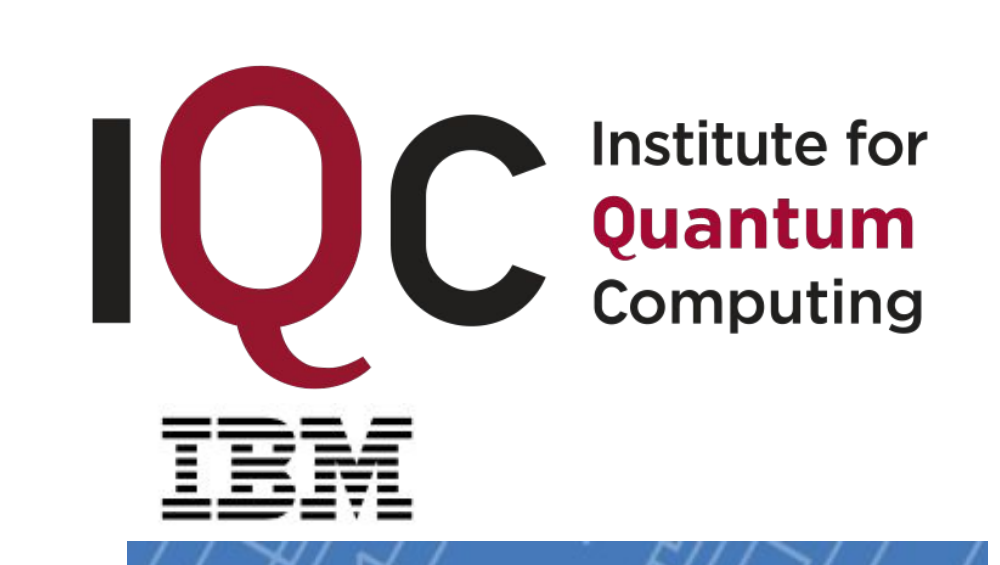

Quantum optimization for LHC physics

- Employ quantum computing to estimate systematics due to Color Recombination models

Solving an optimization problem of phenomenological interest

Formulate the energy minimization as a binary constraint satisfaction problem

- Solve for realistic partonic configurations aiming to find a global minimum

Minimize the color energy subject to constraints.

(

Ealuating the impact on measurements such as top quark mass.

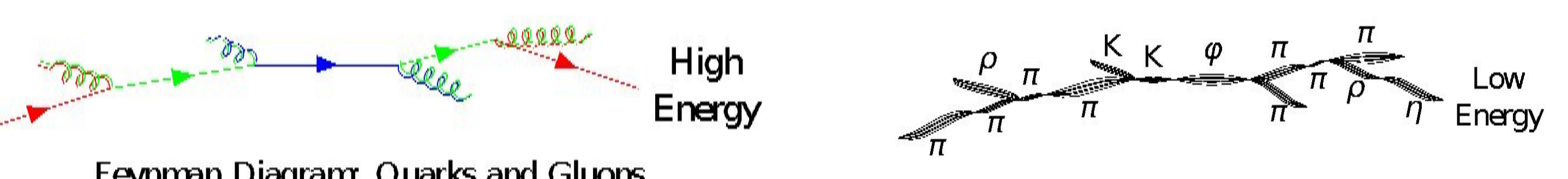

HEP color reconnections in LHC

- Confinement of colored partons into colorless particles

may be described with the Lund string model.

arrangement of quarks and antiquarks, find the

$=\sum_{\substack{i=1 \\ j=i+1}}^{n+1} \ln \frac{m_{i j}^{2}}{m_{0}^{2}}=\prod_{\substack{i=1 \\ j=i+1}}^{n+1} \ln \frac{m_{i j}^{2}}{m_{0}^{2}}$

Problem representation using graph theory

- Nodes are particles and edgies interactions: Quarks a
- nodes 1 Anticuarks are destination nodes

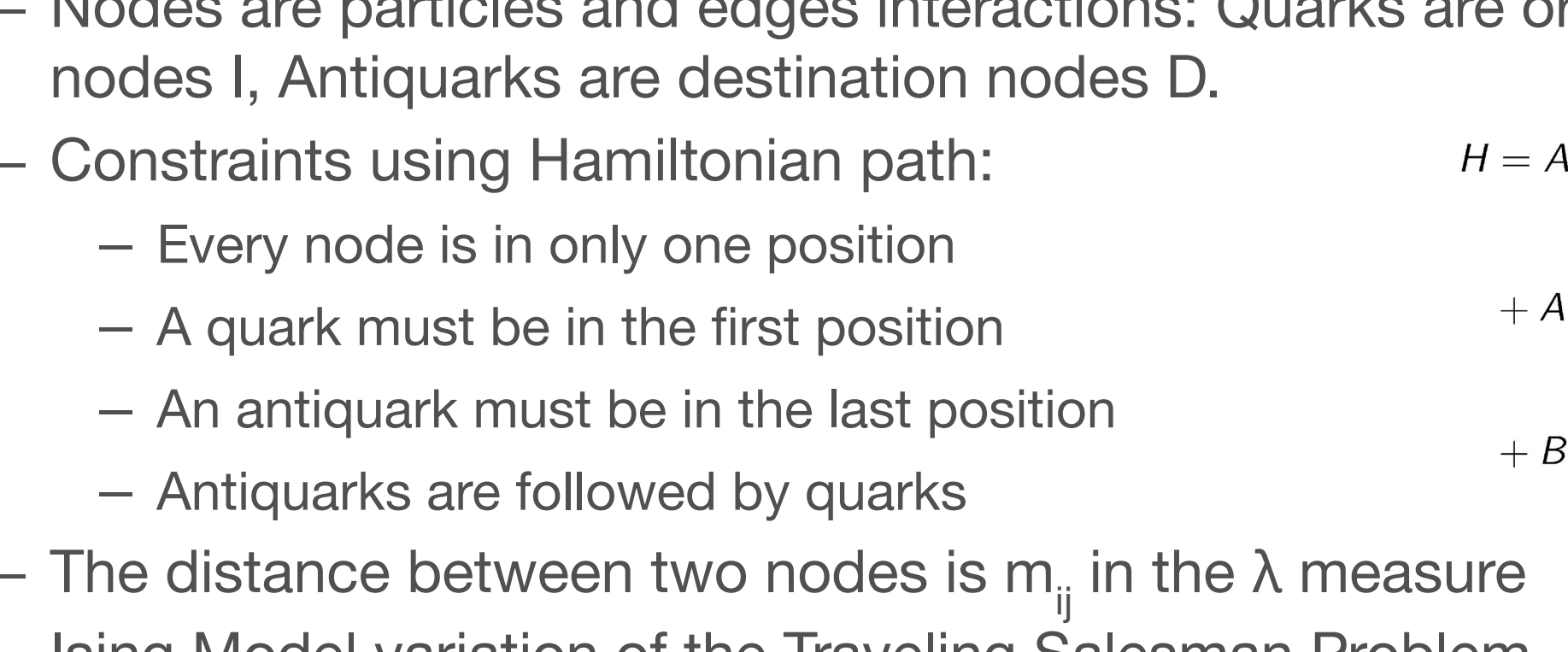

- The distance between two nodes is $m_{i j}$ in the $\lambda$ measu

Connections between particles are nodes, edges form paths

Quarks and antiquarks must have one connection

Start with simplest foptimal path utilizing all nodes

Comparing solvers and accuracy

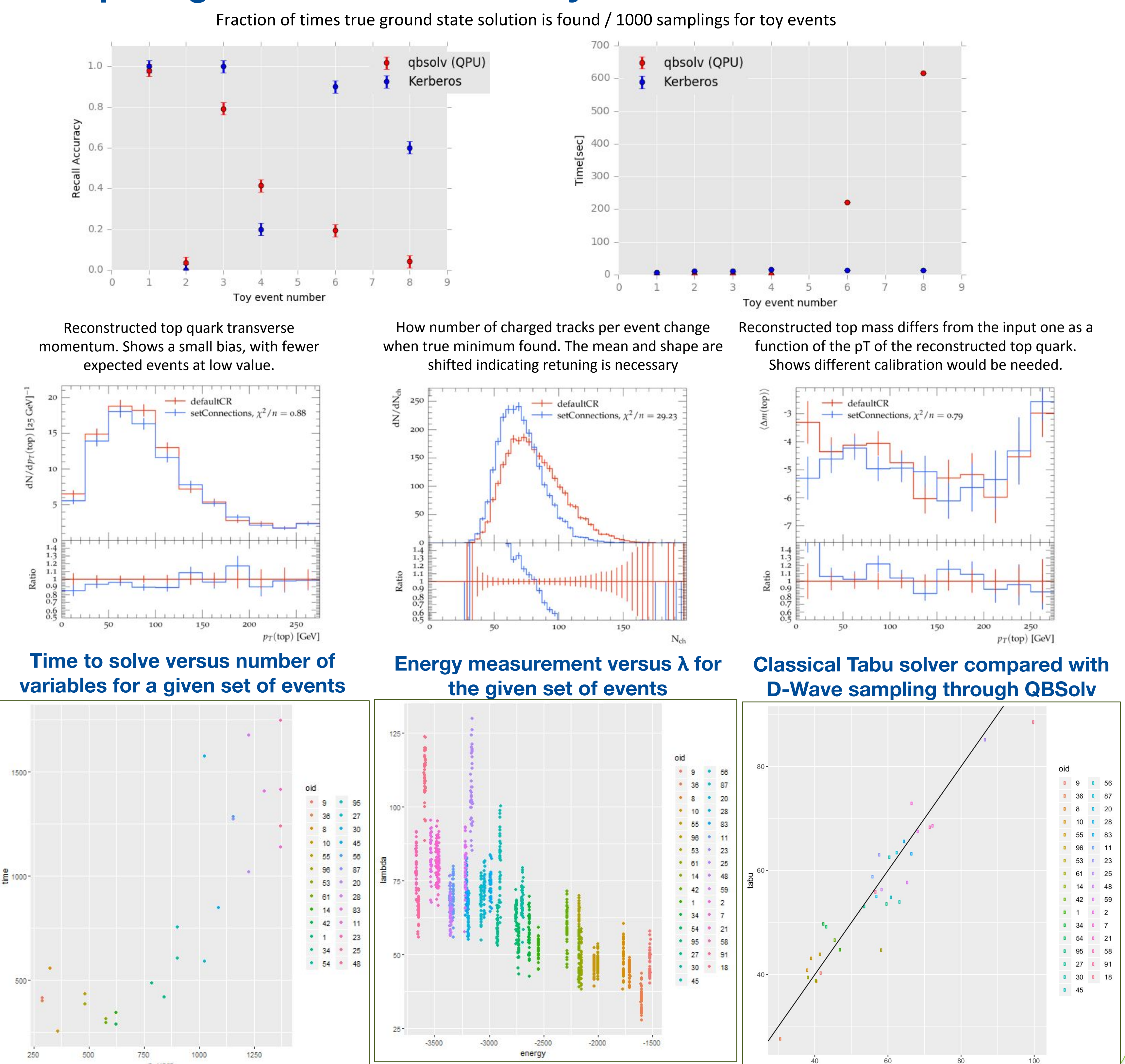

Status

QISKit C. Cirq

- Physics test events generated can be generated on demand

Classical solver and QUBO formulations complete,

Gate-based solution using QAOA working for very small events

Can already run on some platforms

AMPL/CPLEX

This manuscript has been authored by Fermi Research Alliance, LLC under
Contract No. DE-AC02-07CH11359 with the U.S. Department of Energy,
Office of Science, Office of High Energy Physics. This research used
resources of the Oak Ridge Leadership Computing Facility, which is a DOE
Office of Science User Facility supported under Contract
DE-AC05-00OR22725. This work is supported in part by the U.S. Department DE-AC05-00OR22725. This work is supported in part by the U.S.
of Energy, Office of Science, High-Energy Physics Program Office. 